\title{
REGIONALIZACIÓN DEL CAUDAL MÁXIMO ANUAL EN CUENCAS DEL SISTEMA HIDROGRÁFICO DEL RÍO COLORADO, ARGENTINA
}

\author{
CAROLINA LAURO ${ }^{1 *}$, ALBERTO VICH ${ }^{1,2}$, STELLA M. MOREIRAS $^{1,3}$, \\ LUIS BASTIDAS ${ }^{1}$, SEBASTIÁN OTTA ${ }^{1}$, EMILCE VACCARINO ${ }^{1}$
}

${ }^{1}$ Instituto Argentino de Nivología, Glaciología y Ciencias Ambientales, CCT-Mendoza, CONICET, Argentina.

${ }^{2}$ Instituto de Estudios del Ambiente y los Recursos Naturales (IDEARN), Facultad de Filosofía y Letras, Universidad Nacional de Cuyo, Argentina.

${ }^{3}$ Cátedra de Edafología, Facultad de Ciencias Agrarias, Universidad Nacional de Cuyo, Argentina.

\begin{abstract}
RESUMEN. La predicción del caudal máximo anual resulta de utilidad para la gestión de eventos extremos relacionados con crecidas. Para realizar estimaciones con bajo grado de incertidumbre es necesario contar con una gran cantidad de información hidrométrica. Las cuencas que integran el sistema hidrográfico del río Colorado presentan una topografía que dificulta el mantenimiento de las estaciones hidrométricas, razón por la cual, se carece de continuidad en los registros y se presentan en varios casos sectores sin aforar. La regionalización consiste en la transferencia de información de los sitios aforados a los no aforados para la predicción de caudales. El objeto del trabajo fue encontrar la función que relaciona el caudal máximo anual con las características climáticas y morfométricas de las cuencas. Para ello, se emplearon modelos de regresión simple. A partir de dicha relación y la curva de frecuencia regional es posible predecir caudales máximos anuales para diferentes períodos de retorno en cuencas sin aforo pertenecientes al sistema hidrográfico del río Colorado, Argentina. De los doce modelos analizados, los mejores resultados se presentan cuando la variable predictora es el área y perímetro de la cuenca. Los errores de los modelos de regionalización para diversos sitios del sistema resultaron entre el $6 \%$ y el $67 \%$. Estos modelos constituyen una herramienta para la gestión de crecidas en el centro oeste argentino.
\end{abstract}

\section{Annual maximum flow regionalization of Colorado River System, Argentina}

\begin{abstract}
The prediction of the maximum annual flow is necessary for flood management. Large amounts of hydrological information are required to make meaningful estimates. The Colorado River System basins have a topography that makes it difficult to maintain hydrometric stations, so there is a lack of continuity in records and in several cases there are ungauged basins. Regionalization methods consist of transferring information from gauged to ungauged sites in order to make predictions. The objective is to find regional regression models that relate the climate and morphometric characteristics of the basins with the maximum annual flow. For this purpose simple linear regression models were used. From this relationship and the regional frequency curve it will be possible to predict the maximum annual flows for different return periods in ungauged basins of the Colorado River System, Argentina. Regionalization models show that the best estimates occur when the predictor variable is the area and perimeter of the basin. Errors in the regionalization models of various sites in the system resulted between $6 \%$ and $67 \%$. The models found are a tool for flood management in central-western Argentina.
\end{abstract}


Palabras clave: Regionalización, crecida, modelos de regresión, período de retorno.

Keywords: Regionalization, flood, regression models, return period.

Recibido: 26 Diciembre 2019

Aceptado: 16 Septiembre 2020

*Correspondencia: Carolina Lauro, Instituto Argentino de Nivología, Glaciología y Ciencias Ambientales, CCTMendoza, CONICET, Argentina. E-mail: clauro@mendoza-conicet.gob.ar

\section{Introducción}

La predicción de caudales es de utilidad para la planificación de la gestión de los recursos hídricos, por ejemplo, el diseño de la infraestructura hídrica, restauración de ríos, determinación de caudales ambientales y gestión de riesgos, entre otros. Particularmente, la predicción de caudales máximos resulta de gran importancia para la prevención y mitigación de los impactos de desastres naturales asociados a las crecidas e inundaciones. Además, la correcta estimación de la magnitud y frecuencia de los eventos hidrológicos es un factor limitante en la viabilidad económica de obras hidráulicas y gestión de los recursos hídricos.

La manera directa de estudiar el comportamiento hidrológico de una cuenca es a través del análisis de series de datos temporales de caudales disponibles para el sitio de interés. Cuando la información hidrométrica no está disponible o la longitud temporal de los registros no es la apropiada para realizar extrapolaciones a largo plazo, se debe recurrir a métodos indirectos para estudiar las características hidrológicas de la cuenca. Estos métodos consisten en transferir información de sitios donde existe información hidrométrica hacia sitios donde no existen tales registros. Se basan en el principio de compensar la ausencia de datos temporales a través de la abundancia espacial (Hosking y Wallis, 1997).

En la región de Los Andes Centrales, la escasez de sitios de observación de variables hidrológicas y la discontinuidad en las mediciones sistemáticas incrementan la dificultad en la obtención de información hidrológica de base. Ante esta problemática, resulta necesario buscar formas de transferencia de información de un sitio a otro en una cuenca o región (Vich et al., 2014; Lauro et al., 2018).

En los últimos años se ha avanzado en la exploración y aplicación de nuevas herramientas matemáticas, estadísticas y computacionales para la evaluación de los recursos hídricos utilizando toda la información disponible en una región (Poveda et al., 2002; Blöschl et al., 2013). Por un lado, se puede mencionar el análisis regional de frecuencias, cuya principal ventaja es que permite paliar el déficit temporal de información con la abundancia espacial, aumentando la longitud de los registros disponibles mediante la transferencia de información de los diferentes sitios pertenecientes a una región homogénea (Hosking y Wallis, 1997; Teklu y Knut, 2017). Por otro lado, la regionalización hidrológica, definida como un conjunto de herramientas que a partir del análisis de datos hidrométricos, de variables climáticas y características geológicas, permite estimar la magnitud de las variables hidrológicas en sitios con información insuficiente o sin datos (Franchini y Suppo, 1996; Tucci, 1997; Viglione et al., 2007; Song et al., 2016; Kuzmin et al., 2019). La principal ventaja de los estudios regionales es la de extrapolar o interpolar los parámetros para lugares sin datos, optimizando al máximo la información disponible en la región (Mesa Sánchez et al., 2003).

Éstas son prácticas poco usadas en la región de Los Andes Centrales. Puede mencionarse los trabajos realizados por Vich et al. (2014) y el de Lauro et al. (2018) en la región de Cuyo. Los autores 
realizaron la estimación de cuantiles y de parámetros de las funciones de distribución de frecuencias para diversas variables hidrológicas empleando momentos regionales adimensionales, con el método de momentos-L. Además, encontraron que el análisis de frecuencia regional presenta mejor comportamiento y menor incertidumbre en las estimaciones, principalmente para eventos de baja frecuencia, en comparación con el análisis de frecuencia local (Lauro et al., 2018).

El objetivo de este trabajo es determinar la función que relaciona las características morfométricas y climáticas de las cuencas con el caudal máximo anual. Su finalidad es obtener el factor de escala de la curva de crecimiento regional adimensional, para predecir el caudal máximo para diferentes períodos de retorno en cuencas sin aforo pertenecientes al sistema hidrográfico del río Colorado.

\section{2. Área de estudio}

El sistema hidrográfico del río Colorado en la región centro-oeste de Argentina (Fig.1a) abarca una extensión de más de $360.000 \mathrm{Km}^{2}$. Las corrientes fluyen desde la cordillera de Los Andes hacia el este hasta el colector del río Desaguadero; éste drena en el río Colorado, el cual está formado por la confluencia de los ríos Grande y Barrancas.

El régimen hidrológico es nivo-glaciar, con ocurrencia de caudales máximos en el período primavera-verano, entre los meses de noviembre a febrero (Lauro et al., 2019). La variabilidad interanual y decenal del caudal máximo anual de la región se asocia con el índice climático Niño 3.4 y el índice de la Oscilación Decenal del Pacífico (Lauro et al., 2019).

El análisis de regionalización se realizó considerando 12 cuencas del sistema hidrográfico del río Colorado (Fig.1b). Éstas constituyen una región homogénea, es decir, que los caudales diarios máximos de todos los sitios se ajustan a la función de distribución General de Valores Extremos (GEV), diferenciándose sólo por un factor de escala particular para cada sitio (Lauro et al., 2018). Se considera como factor de escala el valor medio de la serie completa de caudales diarios máximos anuales para cada sitio. Todos los sitios que integran la región homogénea acumulan un total de 567 años de registros. Las características estadísticas de las series para cada sitio se presentan en la Tabla 1. Las series de caudal máximo anual se construyeron a partir de los caudales máximo diario por año, promedio para la longitud registro de la serie. Los datos se obtuvieron de la Base de Datos Hidrológica Integrada de la Secretaría de Infraestructura y Política Hídrica de Argentina.

La temperatura es un parámetro importante para caracterizar el régimen fluvial de las corrientes con nacientes en alta montaña. Su incremento influye en la fusión nivo-glacial. Las temperaturas son menores conforme aumenta la altitud de la cordillera y la latitud. Las temperaturas máximas anuales correspondientes al período de verano se registran en la estación Buta Ranquil en la cuenca del río Colorado. Las temperaturas de invernales son menores en la cuenca del río Tupungato (Tabla 2). Las condiciones climáticas y la topografía del lugar dificultan la instalación y mantenimiento de las estaciones meteorológicas; por ello, los registros instrumentales en la cordillera son escasos.

En la región de Los Andes Centrales, el ciclo de precipitación al este de la cordillera está influido por masas de aire continental que contribuyen a los totales de precipitación de verano (Compagnucci et al., 2002; Masiokas et al., 2006). El patrón de precipitación, con un máximo en invierno en la zona más alta de Los Andes, es una consecuencia de la interacción entre los flujos de humedad provenientes de los centros de acción de latitudes medias del Pacífico Sur, la topografía de Los Andes, la ocurrencia de bajas segregadas durante la estación fría (Garreaud y Fuenzalida, 2007) y el paso de frentes fríos (Seluchi et al., 2006). La precipitación media entre los $32^{\circ} \mathrm{S}-36^{\circ} \mathrm{S}$ y $69^{\circ} \mathrm{O}-68^{\circ} \mathrm{O}$ alcanza los $200-600 \mathrm{~mm}$ anuales (Viale et al., 2019). En la cuenca del río Mendoza, la cobertura nival muestra variaciones estacionales con máximos en invierno que alcanzan el 58\% de cobertura promedio anual y variaciones interanuales que marcan períodos secos donde la cobertura alcanza el 12\%, contra los períodos con 
mayor incidencia de nevadas que cubren el 32\% anual promedio (Cara et al., 2016). La mayor precipitación media anual se registra en la cuenca del río Tunuyán en la estación Valle de Uco, mientras que los registros más bajos pertenecen a la estación Punta de Vacas en la cuenca del río Mendoza (Tabla 2).

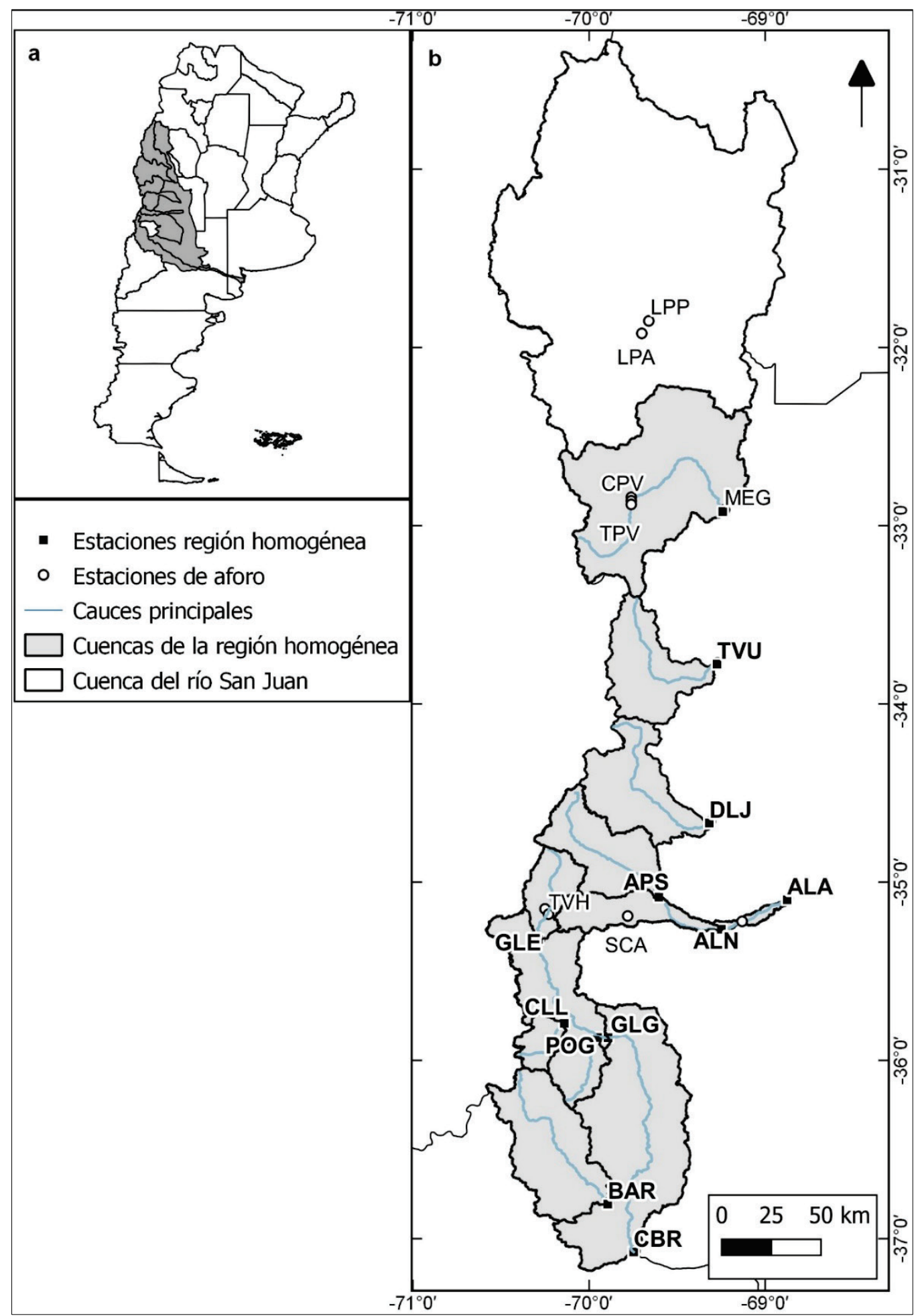

Figura 1. a) Localización regional de las cuencas que integran el sistema hidrográfico del río Colorado. b) Localización de las estaciones de aforo del sistema hidrográfico del río Colorado; sitios que integran la región homogénea y sitios empleados para la predicción. Ver siglas en Tabla 1. 
Tabla 1. Características estadísticas del caudal máximo anual para cada cuenca.

\begin{tabular}{|c|c|c|c|c|c|c|c|c|c|}
\hline Cuenca & Río & Estación & Sigla & Registro & $\begin{array}{c}\text { Caudal } \\
\text { máximo } \\
\text { anual } \\
\left(\mathrm{m}^{3} / \mathbf{s}\right)\end{array}$ & $\begin{array}{c}\text { Máx } \\
\left(\mathbf{m}^{3} / \mathbf{s}\right)\end{array}$ & $\begin{array}{c}\text { Mín } \\
\left(\mathbf{m}^{3} / \mathbf{s}\right)\end{array}$ & $\begin{array}{c}\begin{array}{c}\text { Desvío } \\
\text { estandar } \\
\left(\mathbf{m}^{3} / \mathbf{s}\right)\end{array}\end{array}$ & $\begin{array}{c}\text { Coef. } \\
\text { Variación } \\
(\%)\end{array}$ \\
\hline Mendoza & Mendoza & Guido & MEG & 1956-11 & 149,6 & 401,5 & 51,8 & 73,3 & 49,0 \\
\hline Tunuyán & Tunuyán & Valle de Uco & TVU & 1944-11 & 92,8 & 211,0 & 41,1 & 39,6 & 42,7 \\
\hline Diamante & Diamante & La Jaula & DLJ & 1971-11 & 102,7 & 227,0 & 29,0 & 47,3 & 46,1 \\
\hline \multirow{3}{*}{ Atuel } & Atuel & El Sosneado & APS & 1972-11 & 121,3 & 306,5 & 54,3 & 50,5 & 41,7 \\
\hline & Atuel & Loma Negra & ALN & $1982-11$ & 95,8 & 210,5 & 35,8 & 39,3 & 41,0 \\
\hline & Atuel & $\begin{array}{c}\text { La } \\
\text { Angostura } \\
\end{array}$ & ALA & 1931-11 & 87,8 & 207,5 & 37,0 & 31,5 & 35,8 \\
\hline \multirow{4}{*}{ Grande } & Chico & Las Loicas & CLL & 1991-11 & 56,5 & 128,4 & 8,8 & 26,2 & 2,2 \\
\hline & PotiMalal & $\begin{array}{c}\text { Puesto } \\
\text { Gendarmería }\end{array}$ & POG & 1971-11 & 31,2 & 80,0 & 3,6 & 19,7 & 1,6 \\
\hline & Grande & $\begin{array}{c}\mathrm{La} \\
\text { Estrechura }\end{array}$ & GLE & 1977-11 & 127,8 & 320,0 & 29,3 & 63,2 & 49,4 \\
\hline & Grande & La Gotera & GLG & 1971-11 & 391,5 & 840,0 & 70,5 & 142,8 & 2,7 \\
\hline Barrancas & Barrancas & Barrancas & BAR & 1960-11 & 117,0 & 344,4 & 23,3 & 56,3 & 2,1 \\
\hline Colorado & Colorado & Buta Ranquil & CBR & 1939-11 & 486,7 & $1.404,1$ & 96,1 & 233,1 & 2,1 \\
\hline
\end{tabular}

Tabla 2. Datos de precipitación y temperatura en distintas cuencas del sistema hidrográfico del río Colorado. Los datos se extrajeron de la Base de Datos Hidrológica Integrada de la Secretaría de Infraestructura y Política Hidrica de Argentina.

\begin{tabular}{|c|c|c|c|c|c|c|c|c|c|c|c|}
\hline \multirow{2}{*}{ Cuenca } & \multirow{2}{*}{ Estación } & \multirow{2}{*}{ Sigla } & \multirow{2}{*}{$\begin{array}{c}\text { Elevación } \\
\text { (msnm) }\end{array}$} & \multicolumn{4}{|c|}{ Temperatura $\left({ }^{\circ} \mathrm{C}\right)$} & \multicolumn{4}{|c|}{ Precipitación (mm) } \\
\hline & & & & Serie & Anual & Verano & Invierno & Serie & Anual & Verano & Invierno \\
\hline \multirow{2}{*}{ Mendoza } & Guido & MEG & 1550 & $1994-11$ & 15,0 & 20,9 & 9,0 & $1958-11$ & 197,9 & 79,1 & 38,1 \\
\hline & Punta de Vacas & TPV & 2450 & 1994-11 & 10,9 & 16,5 & 4,9 & 1993-11 & 182,4 & 14,9 & 70,0 \\
\hline Tunuyán & Valle de Uco & TVU & 1200 & 1998-11 & 14,1 & 20,4 & 7,9 & 1955-11 & 419,6 & 133,1 & 89,5 \\
\hline Diamante & La Jaula & DLJ & 1500 & 1994-11 & 13,1 & 19,5 & 6,8 & $1975-11$ & 225,7 & 71,9 & 59,9 \\
\hline Colorado & Buta Ranquil & CBR & 850 & 1994-11 & 16,2 & 23,5 & 9,2 & 1960-11 & 198,0 & 35,1 & 55,1 \\
\hline
\end{tabular}

\section{Materiales y métodos}

\subsection{Obtención de parámetros morfométricos}

Los parámetros morfométricos (Tabla 3) se obtuvieron a partir del modelo digital de elevación (MDE) elaborado por el Instituto Geográfico Nacional (IGN, 2014). Este tiene una resolución de 45x45 $\mathrm{m}$, georreferenciado en coordenadas geográficas y Datum WGS84. Los sectores limítrofes de la cordillera se complementaron con el modelo Shuttle Radar Topography Mission (SRTM) de la NASA. El tamaño de pixel es de 39x39 m, con coordenadas planas UTM (faja 19S). Los procedimientos se realizaron con el software QGIS y planillas de cálculo.

La red hídrica considerada en este trabajo es la correspondiente a las cartas topográficas 1:250.000 del Instituto Geográfico Nacional. 
Tabla 3. Parámetros morfométricos que se utilizaron en el estudio.

\begin{tabular}{|c|c|c|c|c|}
\hline & Parámetro & Unidad & Ecuación/Definición & Referencia \\
\hline \multirow{4}{*}{ 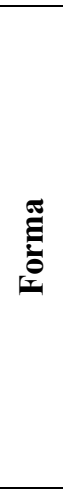 } & Área(A) & $\mathrm{km}^{2}$ & $\begin{array}{c}\text { Área resultante de la proyección de la cuenca en un } \\
\text { plano. }\end{array}$ & \\
\hline & Perímetro(P) & $\mathrm{km}$ & $\begin{array}{l}\text { Longitud del contorno de la proyección de la cuenca en } \\
\text { un plano. }\end{array}$ & \\
\hline & $\begin{array}{c}\text { Relación de } \\
\text { circularidad (C) }\end{array}$ & --- & $\begin{array}{c}C=\frac{4 \pi A_{u}}{P_{u}^{2}} \text { Relaciona el área de la cuenca con la } \\
\text { superficie de un círculo que posee igual perímetro que } \\
\text { la unidad de estudio. }\end{array}$ & Miller, 1953 \\
\hline & $\begin{array}{l}\text { Relación de } \\
\text { elongación (RE) }\end{array}$ & --- & $\begin{array}{c}E=\frac{\sqrt{\frac{4 A_{u}}{\pi}}}{L} \text { Es la relación entre el diámetro de un círculo } \\
\text { con igual área que la de la cuenca y la longitud máxima } \\
\text { de la misma. }\end{array}$ & Schumm, 1956 \\
\hline \multirow{8}{*}{ 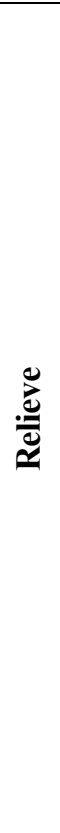 } & Cota máxima(Hmax) & msnm & $\begin{array}{c}\text { Altura máxima de la cuenca. Valor máximo del pixel } \\
\text { correspondiente a la cuenca. }\end{array}$ & \\
\hline & Cota mínima(Hmin) & msnm & $\begin{array}{l}\text { Altura mínima de la cuenca. Valor mínimo del pixel } \\
\text { correspondiente al MDE de la cuenca. }\end{array}$ & \\
\hline & $\begin{array}{l}\text { Diferencia cota } \\
\text { máxima y mínima } \\
\text { (DH) }\end{array}$ & msnm & $\begin{array}{l}\text { Diferencia entre altura máxima y mínima. Diferencia } \\
\text { entre los valores de pixel máximo y mínimo del MDE } \\
\text { de cada cuenca. }\end{array}$ & \\
\hline & Cota media (Hm) & $\mathrm{msnm}$ & Valor medio de las alturas en la cuenca. & \\
\hline & $\begin{array}{l}\text { Pendiente media de la } \\
\text { cuenca (S) }\end{array}$ & $\%$ & Pendiente promedio de todas las laderas. & \\
\hline & $\begin{array}{c}\text { Longitud del cauce } \\
\text { principal (LCP) }\end{array}$ & $\mathrm{km}$ & $\begin{array}{l}\text { Longitud del cauce que conecta el punto de aforo con el } \\
\text { punto más alejado de la cuenca. }\end{array}$ & \\
\hline & $\begin{array}{c}\text { Longitud total de } \\
\text { cauces }(\mathbf{L C T})\end{array}$ & $\mathrm{km}$ & Longitud de todos los cauces presentes en la cuenca. & Linsley et al., 1977 \\
\hline & $\begin{array}{c}\text { Tiempo de } \\
\text { Concentración (Tc) }\end{array}$ & hs & $\begin{array}{l}\text { Tiempo en el cual la escorrentía superficial del punto } \\
\text { más alejado de la cuenca alcanza el punto de desagüe. } \\
\text { Donde J es la pendiente del cauce principal. } \\
\qquad T C=0,3\left(\frac{L C P}{J^{0,25}}\right)^{0,76}\end{array}$ & Témez, 1987 \\
\hline 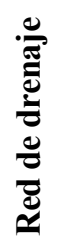 & $\begin{array}{l}\text { Densidad de drenaje } \\
\text { (DD) }\end{array}$ & $\mathrm{km} / \mathrm{km}^{2}$ & $\begin{array}{l}D_{d}=L_{t} / A_{u} \text { Relación entre la longitud de todos los } \\
\text { cauces de cualquier orden (Lt) y el área de la cuenca. }\end{array}$ & Horton 1932 \\
\hline
\end{tabular}

\subsection{Obtención de variables climáticas: precipitación y temperatura}

Debido a baja cantidad de registros instrumentales en la región, se emplearon datos de precipitación y temperatura pertenecientes a la base de datos del Climate Research Unit (CRU) TS4.03 (Harris et al., 2014), expuestos como datos grillados mensuales con resolución espacial de $0,5^{\circ} \times 0,5^{\circ}$. Esta base de datos representa de forma adecuada las variaciones climáticas en la región de Los Andes Centrales (Rusticucci et al., 2014).

Para cada una de las cuencas se obtuvo la precipitación y temperatura media anual para el período 1950-2011. El procedimiento se basó en la obtención de promedios ponderados entre las variables climáticas consideradas, de acuerdo a la superficie de cada cuenca, es decir, la sumatoria de los productos entre los valores de las celdas involucradas, por las superficies relativas de estas (en función del área de la cuenca en cuestión). 
Para evaluar el grado de asociación entre el dato grillado y los datos instrumentales, se calculó el coeficiente de correlación para el período común entre ambas bases de datos.

\subsection{Relación entre parámetros morfométricos y variables climáticas}

En primer lugar, se realizó un análisis de correlación para encontrar los parámetros que poseen mayor relación con la variable dependiente con la intención de reducir la cantidad de variables predictoras a analizar.

La regionalización de caudales se realizó a través de un análisis de regresión simple, donde se considera el caudal máximo promedio como variable respuesta (dependiente) y los parámetros morfométricos y las variables climáticas como variables predictoras (independientes). La estimación de los parámetros del modelo se realizó por medio de mínimos cuadrados ordinarios. Se emplearon tres tipos de modelos: lineal, potencial y exponencial. Mediante las transformaciones correspondientes los modelos potenciales y exponenciales fueron linealizados.

La eficacia de los modelos se evaluó por medio del coeficiente de determinación $\left(\mathrm{R}^{2}\right)$ y el error cuadrático medio (RMSE) cuantificado en las mismas unidades que la variable respuesta $\left(\mathrm{m}^{3} / \mathrm{s}\right)$. Además, se calculó el porcentaje de error aproximado del modelo, dividiendo el valor de RMSE por el valor promedio de la variable respuesta.

\subsection{Aplicación de los modelos}

Los modelos regionales de regresión se aplicaron para calcular el caudal máximo anual de diversas cuencas cuyo caudal máximo anual es conocido con la finalidad de evaluar la capacidad predictiva de los mismos. Los sitios empleados pertenecen a cuencas sistema hidrográfico del río Colorado (Fig. 1b).

A partir de los valores medios obtenidos con los modelos de regresión y la curva de frecuencia regional (Lauro et al., 2018) se calcularon los caudales máximo anuales para diferentes períodos de retorno.

\section{Resultados}

\subsection{Características morfométricas y climáticas de la región homogénea}

En la Tabla 4 se muestran los valores medios, máximos, mínimos y desviación estándar de los parámetros morfométricos y climáticos estimados para las 12 cuencas de la región homogénea.

El caudal anual máximo promedio para la región integrada por 12 cuencas es de $155,1 \mathrm{~m}^{3} / \mathrm{s}$. Los valores mínimos y máximos corresponden a las cuencas de los ríos Potimalal y Colorado, respectivamente.

El promedio del área de las cuencas que integran la región es de $4.033,1 \mathrm{~km}^{2}$, siendo las cuencas del río Chico y del río Colorado las de menor y mayor extensión respectivamente. Los bajos valores del índice de circularidad y el índice de elongación indican que las cuencas se alejan de la forma de círculo, presentando formas ovalo-oblonga a rectangular-oblonga.

En la región de estudio, la elevación del terreno (Fig. 2b) disminuye de norte a sur, alcanzando la altitud máxima en la cuenca del río Mendoza y la mínima en la del río Colorado. Las pendientes medias de las cuencas son características de un relieve irregular y muy fuertemente accidentado (Heras, 1972), con un rango entre $25 \%$ y $48 \%$, siendo representativos de las cuencas del río Colorado y Tunuyán respectivamente. En la Figura 2a se presenta el mapa de pendientes de donde se extrajeron los valores promedio para cada cuenca. 
Tabla 4. Características descriptivas de los parámetros morfométricos y climáticos para la región homogénea.

\begin{tabular}{|c|c|c|c|c|c|}
\hline Variable & n & Mínimo & Máximo & Media & Desv. típica \\
\hline $\mathrm{A}\left(\mathrm{km}^{2}\right)$ & 12 & 595,8 & $14.875,2$ & 4033,1 & 3906,8 \\
\hline $\mathrm{P}(\mathrm{km})$ & 12 & 141,4 & 1038,8 & 447,4 & 251,9 \\
\hline $\mathrm{RC}$ & 12 & 0,1 & 0,4 & 0,3 & 0,1 \\
\hline $\mathrm{RE}$ & 12 & 0,5 & 1,0 & 0,7 & 0,1 \\
\hline $\mathrm{H} \mathrm{Max}(\mathrm{m})$ & 12 & 3807,0 & 6903,0 & 5034,8 & 853,2 \\
\hline $\mathrm{H} \mathrm{Min}(\mathrm{m})$ & 12 & 815,0 & 1994,0 & 1390,0 & 303,4 \\
\hline $\mathrm{Dh}(\mathrm{m})$ & 12 & 2233,0 & 5493,0 & 3644,8 & 941,6 \\
\hline $\mathrm{H} \mathrm{m}(\mathrm{m})$ & 12 & 2169,0 & 3650,0 & 2793,8 & 467,0 \\
\hline $\mathrm{S} \%$ & 12 & 25,0 & 48,0 & 34,0 & 7,0 \\
\hline $\mathrm{LCP}(\mathrm{km})$ & 12 & 42,2 & 365,3 & 143,4 & 87,0 \\
\hline $\mathrm{LCT}(\mathrm{km})$ & 12 & 205,1 & 3542,8 & 1111,0 & 988,0 \\
\hline $\mathrm{Tc}(\mathrm{hs})$ & 12 & 10,4 & 66,5 & 28,9 & 15,38 \\
\hline $\mathrm{DD}\left(\mathrm{km} / \mathrm{km}^{2}\right)$ & 12 & 0,2 & 1,4 & 0,4 & 0,3 \\
\hline $\mathrm{PPM}(\mathrm{mm})$ & 12 & 353,9 & 539,7 & 458,8 & 61,5 \\
\hline $\mathrm{TM}\left({ }^{\circ} \mathrm{C}\right)$ & 12 & 1,8 & 8,3 & 5,6 & 2,2 \\
\hline $\mathrm{QMAX}\left(\mathrm{m}^{3} / \mathrm{s}\right)$ & 12 & 31,2 & 486,7 & 155,1 & 137,8 \\
\hline
\end{tabular}

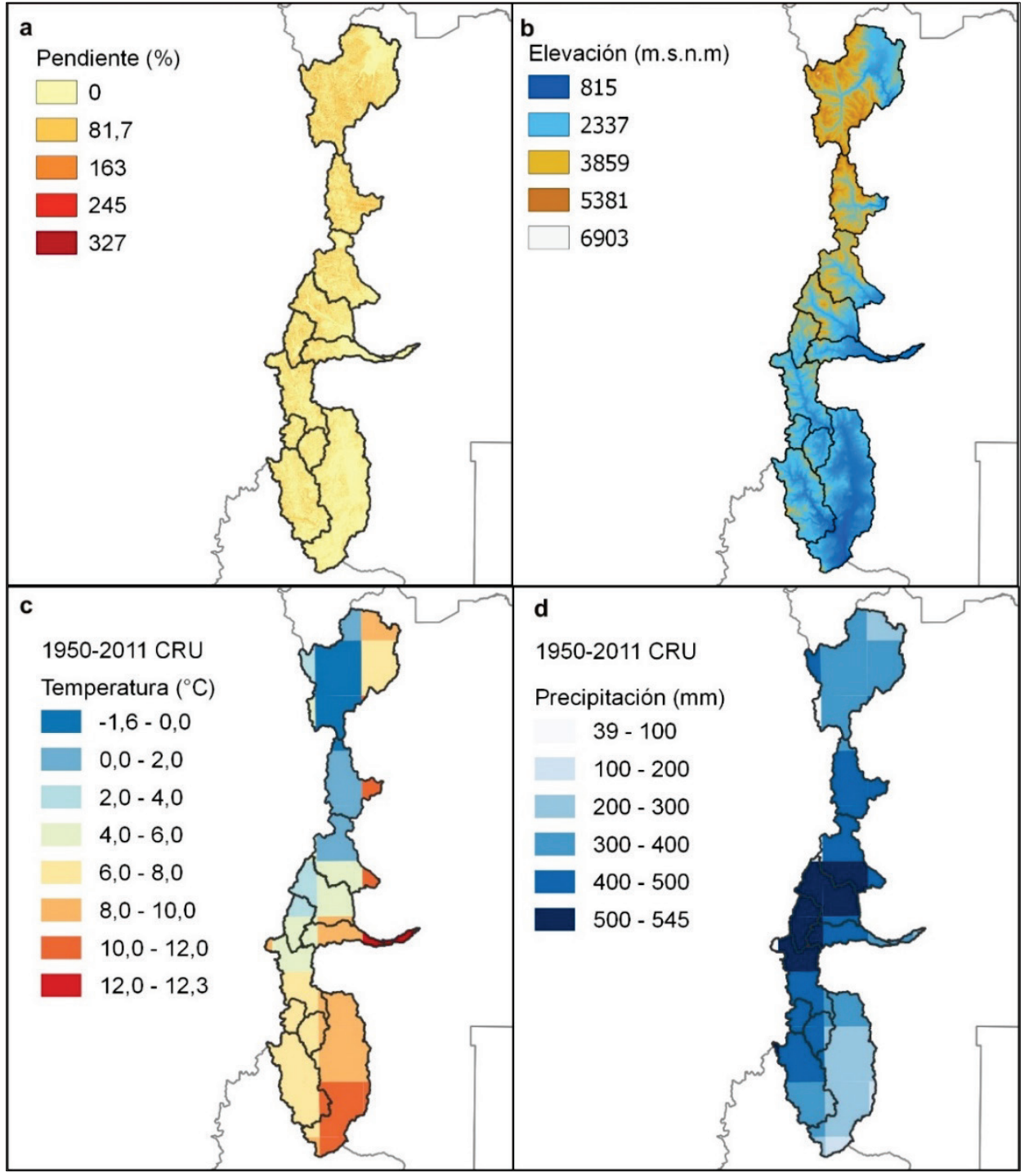

Figura 2. a) Mapas de pendientes (\%), b) elevación (msnm), c) temperatura media anual $\left({ }^{\circ} \mathrm{C}\right), d$ ) precipitación media anual $(\mathrm{mm})$ para la región homogénea. 
En promedio, la longitud del cauce principal y la longitud de todos los cauces es de 143,4 km y $1111 \mathrm{~km}$ respectivamente; en ambos casos, los valores mínimos corresponden a la cuenca del río Chico y los máximos a la cuenca del río Colorado. Las densidades de drenajes son bajas, lo cual es característico de cuencas de zona de montaña con alta permeabilidad.

El tiempo de concentración se encuentra entre 10,04 horas y 66,5 horas correspondientes a las cuencas del río Chico y río Colorado respectivamente.

En líneas generales, la temperatura aumenta hacia el sur y hacia el este de las cuencas conforme disminuye la elevación (Fig. 2c). El promedio de la temperatura media anual de las 12 cuencas es de $5,6^{\circ} \mathrm{C}$, con un rango de $1,8^{\circ} \mathrm{C}$ a $8,3^{\circ} \mathrm{C}$ correspondientes a las cuencas del río Tunuyán y Colorado respectivamente. Dichos valores son inferiores a los procedentes de registros instrumentales (ver Tabla 2) debido a que las estaciones meteorológicas se encuentran en la parte más baja de la cuenca. Los coeficientes de correlación entre la base de datos grillada y los datos instrumentales de temperatura resultaron significativos, con coeficientes entre 0,5 y 0,62 .

Los valores máximos de precipitación se encuentran en la zona central de la región analizada, en las cuencas del río Atuel y río Grande, dejando a los valores mínimos en los extremos norte y sur correspondientes a las cuencas del Mendoza y Colorado (Fig.2d). Los coeficientes de correlación entre los datos instrumentales y grillados de precipitación resultaron significativos, con magnitudes entre 0,42 y 0,75 .

\subsection{Análisis de correlación}

El caudal máximo anual se correlaciona positiva y significativamente con el área, el perímetro, la longitud del cauce principal, la longitud de todos los cauces y el tiempo de concentración (Tabla 5). A su vez, estas variables tienen correlaciones significativas entre ellas, por lo que incorporarlas todas juntas en un modelo de regresión múltiple no generaría aportaciones sustanciales al modelo, pudiendo ocasionar efecto de co-linealidad. La multicolinealidad trae como consecuencia errores en la interpretación de la contribución individual de cada parámetro (Costa, 2017). Para la elección del modelo, se sugiere considerar un número veraz de parámetros de regresión, a fin de garantizar la fiabilidad y solidez del modelo estadístico, considerando el principio de parsimonia (Noto y La Loggia, 2009; Cassahlo, 2018). 


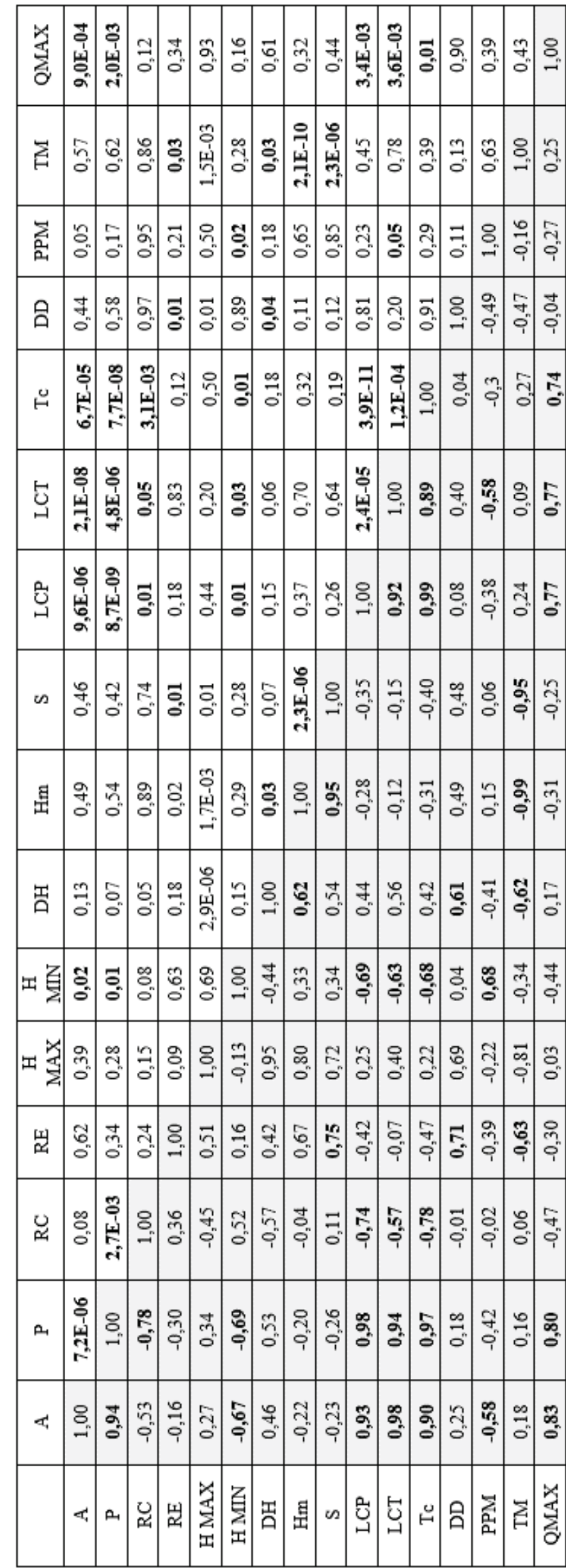

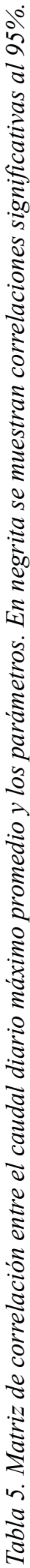




\subsection{Análisis de regresión}

Dada la poca cantidad de sitios que integran la región homogénea $(\mathrm{n}=12)$, no es conveniente utilizar más de un parámetro por modelo para evitar efectos de colinealidad y sobreparametrización, como se mencionó anteriormente. Por ello, se realizaron regresiones lineales con las cinco variables predictoras que tenían mayor correlación con la variable respuesta. Las variables predictoras son: área, perímetro, longitud del cauce principal, longitud total de todos los cauces que integran la cuenca y el tiempo de concentración.

Tras comparar los modelos, el modelo lineal presentó el coeficiente de determinación mayor y el menor RSME en todos los casos, excepto para el modelo exponencial con la variable perímetro, donde el RSME es levemente inferior (Tabla 6).

Tabla 1. Resumen de las características estadísticas de los modelos obtenidos para cada uno de los parámetros (A, área; P, perímetro; LCP, longitud cauce principal; LCT, longitud total de cauces).

\begin{tabular}{|c|c|c|c|}
\hline & Modelo Lineal & Modelo Potencial & Modelo exponencial \\
\hline $\mathbf{A}$ & 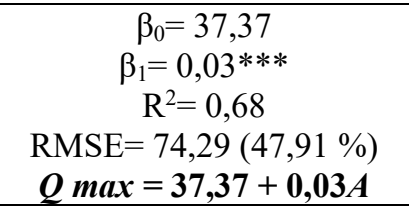 & $\begin{array}{c}\beta_{0}=-0,17 \\
\beta_{1}=0,65^{* *} \\
\mathrm{R}^{2}=0,62 \\
\mathrm{RMSE}=83,93(54,13 \%) \\
\boldsymbol{Q} \boldsymbol{\operatorname { m a x }}=\mathbf{0 , 6 7} \boldsymbol{A}^{\mathbf{0 , 6 5}}\end{array}$ & $\begin{array}{c}\beta_{0}=4,18 * * * \\
\beta_{1}=1,5 \mathrm{E}-04 * * \\
\mathrm{R}^{2}=0,59 \\
\mathrm{RMSE}=79,85(51,5 \%) \\
\boldsymbol{Q} \max =\mathbf{6 5 , 6} \boldsymbol{e}^{A 1,4 E-\mathbf{0 4}}\end{array}$ \\
\hline $\mathbf{P}$ & $\begin{array}{c}\beta_{0}=-39,68 \\
\beta_{1}=0,44 * * * \\
\mathrm{R}^{2}=0,63 \\
\mathrm{RMSE}=79,98(51,58 \%) \\
\boldsymbol{Q} \boldsymbol{m a x}=\mathbf{- 3 9 , 6 8}+\mathbf{0 , 4 4 P}\end{array}$ & $\begin{array}{c}\beta_{0}=-0,33 \\
\beta_{1}=0,93 * * \\
\mathrm{R}^{2}=0,62 \\
\mathrm{RMSE}=90,62(58,45 \%) \\
\boldsymbol{Q} \boldsymbol{m a x}=\mathbf{0 , 4 7 P} \mathbf{0 , 9 3}\end{array}$ & $\begin{array}{c}\beta_{0}=3,74 * * * \\
\beta_{1}=2,3 \mathrm{E}-03 * * \\
\mathrm{R}^{2}=0,62 \\
\mathrm{RMSE}=73,78(47,58 \%) \\
\boldsymbol{Q} \boldsymbol{\operatorname { m a x }}=\mathbf{4 2} \boldsymbol{e}^{\boldsymbol{P 2 . 3 E - 0 3}}\end{array}$ \\
\hline LCP & $\begin{array}{c}\beta_{0}=-19,97 \\
\beta_{1}=1,22 * * * \\
\mathrm{R}^{2}=0,59 \\
\mathrm{RMSE}=84,12(54,25 \%) \\
\boldsymbol{Q} \boldsymbol{\operatorname { m a x }}=\mathbf{- 1 9 , 9 7 + 1 , 2 2 L \boldsymbol { C P }}\end{array}$ & $\begin{array}{c}\beta_{0}=0,13 \\
\beta_{1}=0,93 * * \\
\mathrm{R}^{2}=0,56 \\
\mathrm{RMSE}=91,49(59 \%) \\
\boldsymbol{Q} \boldsymbol{m a x}=\mathbf{1 , 3 5 L C P} \mathbf{C , 9 3}\end{array}$ & $\begin{array}{c}\beta_{0}=3,86 * * * \\
\beta_{1}=0,006^{* *} \\
\mathrm{R}^{2}=0,55 \\
\mathrm{RMSE}=84,63(54,58 \%) \\
\boldsymbol{Q} \max =\mathbf{4 7 , 4} \boldsymbol{e}^{\boldsymbol{L C P \boldsymbol { 0 } , 0 1}}\end{array}$ \\
\hline LCT & $\begin{array}{c}\beta_{0}=36,24 \\
\beta_{1}=0,11 * * * \\
\mathrm{R}^{2}=0,59 \\
\mathrm{RMSE}=84,83(54,71 \%) \\
\boldsymbol{Q} \boldsymbol{m a x}=\mathbf{3 6 , 2 4}+\mathbf{0 , 1 1} \boldsymbol{L C T}\end{array}$ & $\begin{array}{c}\beta_{0}=0,28 \\
\beta_{1}=0,62 * * \\
\mathrm{R}^{2}=0,57 \\
\mathrm{RMSE}=93,84(60,52 \%) \\
\boldsymbol{Q}_{\boldsymbol{m a x}}=\mathbf{1 , 9 0} \boldsymbol{L} \boldsymbol{C} \boldsymbol{T}^{\mathbf{0 , 6 2}}\end{array}$ & 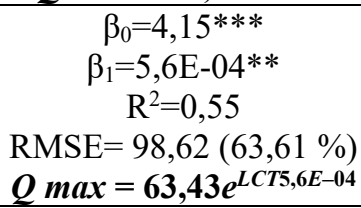 \\
\hline Te & $\begin{array}{c}\beta_{0}=-37,75 \\
\beta_{1}=6,6^{* *} \\
\mathrm{R}^{2}=0,55 \\
\mathrm{RMSE}=88,25(56.91 \%) \\
\boldsymbol{Q} \boldsymbol{\operatorname { m a x }}=\mathbf{- 3 7 , 7 5}+\mathbf{6 , 6 T c}\end{array}$ & 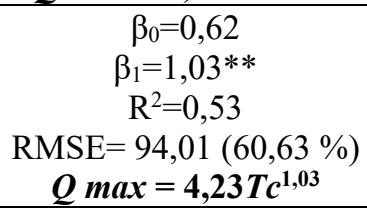 & $\begin{array}{c}\beta_{0}=3,76^{* * *} \\
\beta_{1}=0,035^{* *} \\
\mathrm{R}^{2}=0,52 \\
\mathrm{RMSE}=98,52(63,54 \%) \\
\boldsymbol{Q} \max =\mathbf{4 3 , 2 1} \boldsymbol{e}^{\boldsymbol{T c 0 , 0 3}}\end{array}$ \\
\hline
\end{tabular}

$0 \leq$ Valor-p $\leq 0,001 * * * ; 0,001<$ Valor-p $\leq 0,01 * * ; 0,01<$ Valor-p $\leq 0,05^{*}$

De los doce modelos obtenidos, el que explica mejor el comportamiento del caudal medio máximo es el modelo lineal, con el área de la cuenca como variable predictora. El modelo es significativo (p-valor 0,0009), el cual explica un 68\% de la variabilidad, con un error residual estándar de 74,29 $\mathrm{m}^{3} / \mathrm{s}$, lo que en términos porcentuales representa el $47,91 \%$.

Alternativamente, el modelo exponencial con el perímetro como variable predictora también presenta similares resultados. El modelo es significativo (p-valor 0,0024 ) pues explica el $62 \%$ de la variabilidad. El error residual estándar es $73,78 \mathrm{~m}^{3} / \mathrm{s}$, lo que en términos porcentuales respecto a la media es del $47,58 \%$.

Por lo general, el área de la cuenca es la variable que presenta mayor relación con el caudal en diversos trabajos (Noto y La Loggia, 2009; Malekinezhad et al., 2011; Lujano et al., 2016, 2017). 
Comúnmente se emplean diversas variables ambientales (como precipitación, temperatura o evaporación); sin embargo, en el presente estudio no se encontró una correlación significativa con las variables climáticas.

\subsection{Aplicación de los modelos}

Con los modelos regionales encontrados se realizaron predicciones para los diversos sitios del sistema hidrográfico del río Colorado que reunían las condiciones límites de los modelos; es decir, que tuvieran un área entre 595,5-14.875,2 $\mathrm{km}^{2}$, un perímetro entre $141,4-1038,8 \mathrm{~km}$ y cuyos caudales fueran conocidos para poder evaluar los modelos. Los detalles de los sitios y resultados de los modelos se detallan a continuación (Tabla 7).

Tabla 7. Aplicación de los modelos regionalizados en diversos ríos.

\begin{tabular}{|c|c|c|c|c|c|c|c|c|c|}
\hline Río & Estación & Sigla & $\begin{array}{c}\text { Qmax } \\
\left(\mathrm{m}^{3} / \mathbf{s}\right)\end{array}$ & $\begin{array}{c}\text { Área } \\
\left(\mathrm{km}^{2}\right)\end{array}$ & $\begin{array}{c}\mathbf{Q} \mathbf{m a x} \\
\mathbf{M} . \\
\mathbf{L i n e a l} \\
\left(\mathbf{m}^{\mathbf{3} / \mathbf{s}}\right)\end{array}$ & $\begin{array}{c}\text { Error } \\
(\%)\end{array}$ & $\begin{array}{c}\text { Perímetro } \\
(\mathrm{km})\end{array}$ & $\begin{array}{c}\widehat{\mathbf{Q} m a x} \mathbf{M} . \\
\mathbf{e x p o n e n c i a l} \\
\left(\mathbf{m}^{\mathbf{3} / \mathbf{s})}\right.\end{array}$ & $\begin{array}{c}\text { Error } \\
(\%)\end{array}$ \\
\hline Los Patos & La Plateada & LPP & 166,6 & 8539,6 & 293,6 & 43,3 & 845,6 & 293,7 & 43,3 \\
\hline Los Patos & Álvarez Condarco & LPA & 89,6 & 3581,1 & 114,8 & 38,1 & 475,8 & 125,5 & 28,6 \\
\hline Cuevas & Punta de vacas & CPV & 21,0 & 667,2 & 57,7 & 63,6 & 151,9 & 59,6 & 64,7 \\
\hline Tupungato & Punta de Vacas & TPV & 85,4 & 1783,5 & 90,9 & 6,0 & 257,7 & 76,0 & 12,4 \\
\hline Salado & Cañada Ancha & SCA & 37,4 & 668,5 & 57,4 & 34,8 & 157,8 & 60,8 & 38,0 \\
\hline Tordillo & Valle Hermoso & TVH & 37,4 & 628,5 & 56,2 & 33,5 & 151,9 & 59,6 & 37,3 \\
\hline
\end{tabular}

En los casos del modelo lineal se sobreestimaron los valores de caudal máximo promedio. La mejor estimación fue para el río Tupungato (TPV), con un error del $6 \%$. El modelo exponencial también sobrestima los valores de caudal, salvo para el sitio TPV, donde se subestima, y en este caso, el modelo también tuvo el menor error de estimación (12\%), aunque no menor que el estimado por el modelo lineal. Para el caso del río Los Patos (LPA), el modelo exponencial presentó menor error que el modelo lineal. Para el mismo río, en la estación La Plateada ambos modelos tuvieron similares resultados. En la Figura 3 se muestran los modelos y las predicciones realizadas.

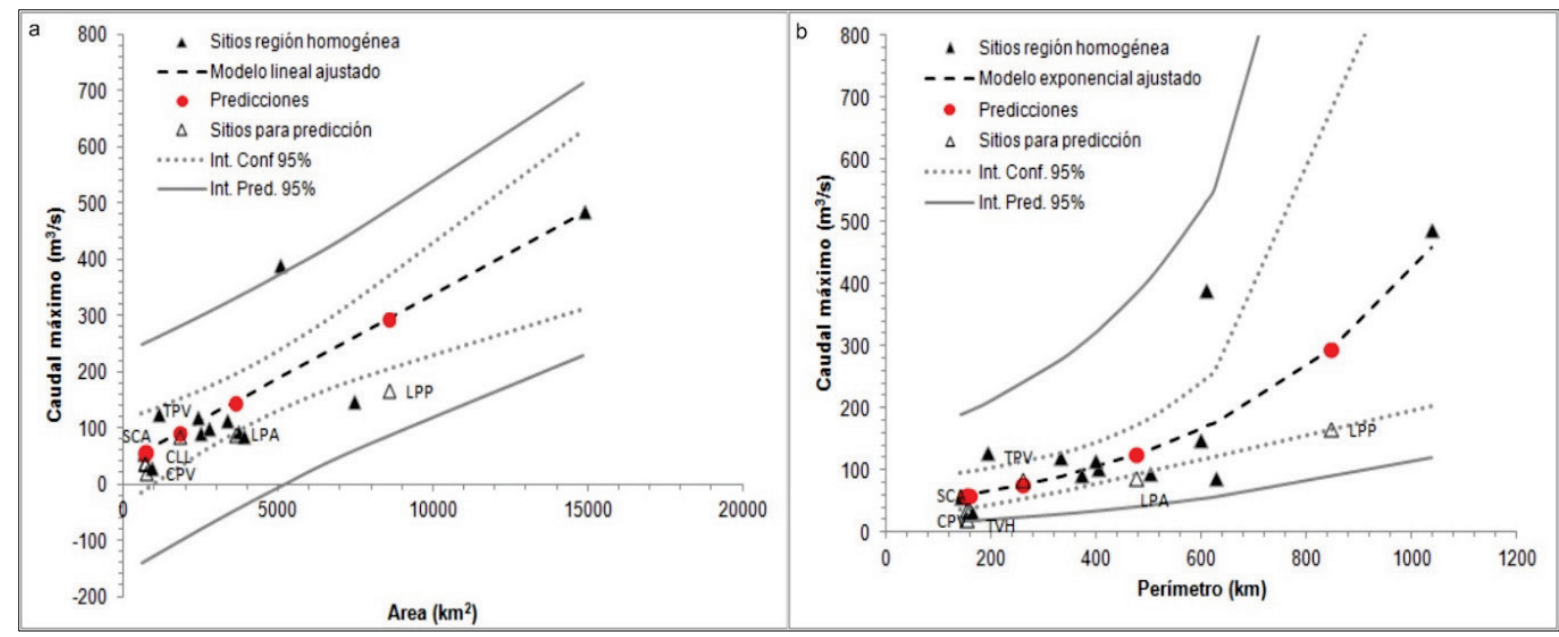

Figura 3. Modelo de regresión lineal (a) y exponencial (b) ajustado a los sitios pertenecientes a la región homogénea. Se muestran los caudales medio máximo anual para seis sitios del sistema del río Colorado y su correspondiente predicción utilizando los modelos de regionalización. 


\subsubsection{Estimación del caudal para diversos períodos de retorno}

Se estimó el caudal máximo para diferentes períodos de retorno (TR), que van desde $\mathrm{TR}=2$ años hasta TR=10.000 años. Para ello se empleó la curva de crecimiento regional adimensional, derivada del análisis de frecuencia regional (Lauro et al., 2018) y los caudales máximos medios obtenidos de las predicciones realizadas con los modelos de regresión. En la Figura 4 se muestran las estimaciones.

Se observa una sobrestimación del caudal cuya menor diferencia se obtuvo para el sitio TPV, con el valor medio del caudal estimado a partir del modelo lineal. Para el sitio LPP se obtuvo la mayor diferencia.

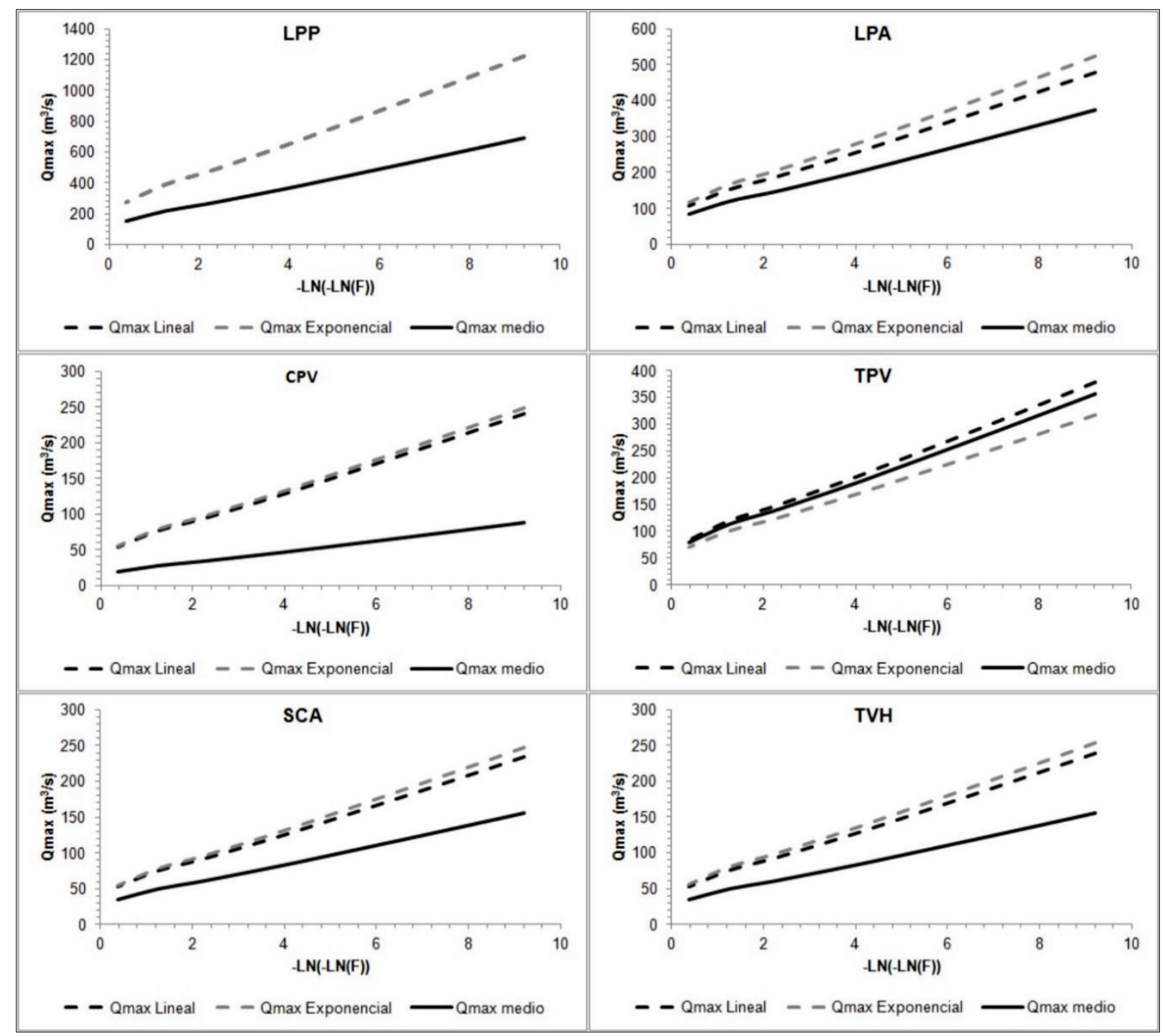

Figura 4. Estimación de caudales máximos para diferentes períodos de retorno.

\section{Conclusiones}

El presente estudio consistió en encontrar modelos regionales que estimasen los caudales máximos anuales en función de las características morfométricas y climáticas de las cuencas que integran el sistema hidrográfico del río Colorado. Estas cuencas poseen la particularidad de presentar sus nacimientos en regiones montañosas de difícil acceso, lo que dificulta el mantenimiento de las estaciones de medición y conduce a la falta de información hidrométrica. 
El bajo número de estaciones hidrométricas que integran la región homogénea condicionó los modelos de regresión a emplear, pudiendo utilizar solo una variable explicativa por regresión, para evitar la sobreparemetrización. Los modelos encontrados permiten obtener el valor de caudal máximo anual en función del área o perímetro de la cuenca. Los modelos son válidos para rangos del área y perímetro para los cuales fueron establecidos y para cuencas en la que se verifique la homogeneidad regional.

Los caudales máximos anuales regionalizados constituyen el factor de escala de la curva regional de frecuencias que fueron empleados para la estimación de caudales para distintos períodos de retorno. En líneas generales se observó una sobreestimación de los caudales, siendo esta mayor para períodos de retornos más grandes.

En futuros estudios deberían considerarse otras variables ambientales que expliquen el comportamiento de los caudales en la región. También deberá contarse con mediciones de campo que permitan verificar la predictibilidad de los modelos.

\section{Referencias}

Blöschl, G., Sivapalan, M., Wagener, T., Viglione, A., Savenije, H. 2013. Runoff prediction in ungauged basins. Synthesis across processes, places and scales. Cambridge University Press. 490 p.

Cara, L., Masiokas, M., Viale, M., Villalba, R. 2016. Análisis de la cobertura nival de la cuenca superior del río Mendoza a partir de imágenes MODIS. Meteorológica 41(1), 21-36.

Cassalho, F., Beskow, S., de Mello, C.R., de Moura, MM. 2018. Regional flood frequency analysis using Lmoments for geographically defined regions: An assessment in Brazil. Journal of Flood Risk Management 12 (2). https://doi.org/10.1111/jfr3.12453

Compagnucci, R.H., Agosta, E.A., Vargas, W.M. 2002. Climatic change and quasi-oscillations in central-west Argentina summer precipitation: main features and coherent behavior with southern African region. Climate Dynamics 18, 421-435. https://doi.org/10.1007/s003820100183

Costa, V. 2017. Correlation and regression. In: M. Naghettini (Ed.), Fundamentals of statistical hydrology (pp. 391-440). Switzerland: Springer. https://doi.org/10.1007/978-3-319-43561-9

Franchini, M., Suppo, M. 1996. Regional analysis of flow duration curves for a limestone region. Water Resources Management 10(3), 199-218. https://doi.org/10.1007/BF00424203

Garreaud, R., Fuenzalida, H. 2007. The Influence of Andes on cutoff lows: A modeling study. Monthly Weather Review 135, 1596-1613. https://doi.org/10.1175/MWR3350.1

Harris, I., Jones, P.D., Osborna, T.J., Lister, D.H. 2014. Updated high-resolution grids of monthly climatic observations-the CRU TS3.10 Dataset. International Journal of Climatology 34 (3), 623-642. https://doi.org/10.1002/joc.3711

Heras, R. 1972. Manual de hidrología. Tomo IV: hidrología de las crecidas. Servicio de Edición del Centro de Estudios Hidrográficos y Dirección General de Obras Hidráulicas.

Horton R.E. 1932. Drainage-Basin characteristics. EOS, Transactions American Geophysical Union. 13 (1), $350-$ 361. https://doi.org/10.1029/TR013i001p00350

Hosking, J., Wallis, J. 1997. Regional Frequency Analysis. An approach based on L-Moments. Cambridge University Press. U K. 240 p.

IGN. 2014. Modelo Digital de elevaciones de la República Argentina MDE-Ar (2014). http://www.ign.gob.ar/NuestrasActividades/Geodesia/ModeloDigitalElevaciones/Mapa

Kuzmin, V., Pivovarova, I., Shemanaev, K., Sokolova, D., Batyrov, A., Tran, N., Dang D. 2019. Method of Prediction of the Stream Flows in Poorly Gauged and Ungauged Basins. Journal of Ecological Engineering 20(1), 180-187. https://doi.org/10.12911/22998993/94915. 
Lauro, C., Vich, A., Moreiras, S.M. 2018. Regional flood frequency analysis in the Central Western River Basins $\left(28^{\circ}-37^{\circ} \mathrm{S}\right)$ of Argentina. River Research and Applications 34 (7), 721-733. https://doi.org/10.1002/rra.3319

Lauro, C., Vich, A., Moreiras, S.M. 2019. Streamflow variability and its relationship with climate indexes in western river basins of Argentina. Hydrological Science Journal 57 (1). http://doi.org/10.1080/02626667.2019.1594820

Linsley, R.K., Kholer, M.A., Paulus, J.L.H. 1977. Hidrología para ingenieros. McGraw-Hill Latinoamericana. Bogotá. 398 p.

Lujano, A., Quispe, J., Lujano, E., Tapia, B. 2017. Regionalización de caudales mensuales en la región hidrográfica del Tititaca, Perú. Revista Investigaciones Altoandinas 19 (2), 219-230. http://doi.org/10.18271/ria.2017.281

Lujano, A., Lujano, E., Quispe, J.P. 2016. Regionalización de caudales anuales en cuencas del altiplano peruano. Revista de Investigaciones Altoandinas 18(2), 189-194. http://doi.org/10.18271/ria.2016.199

Malekinezhad, H., Nachtnebel, H.P., Klik, A. 2011. Comparing the index-flood and multiple-regression methods using L-moments. Physics and Chemistry of the Earth 36, 54-60. https://doi.org/10.1016/j.pce.2010.07.013

Masiokas, M., Villalba, R., Luckman, B., Le Quesne, C., Aravena, J.C. 2006. Snowpack variations in the Central Andes of Argentina and Chile, 1951-2005: Large-scale atmospheric influences and implications for water resources in the region. Journal of Climate 19, 6334-6352. https://doi.org/10.1175/JCLI3969.1

Mesa, Ó.J., Vélez, J.I., Giraldo, J.D., Quevedo, D.I. 2003. Regionalización de características medias de la cuenca con aplicación en la estimación de caudales máximos. Meteorológica Colombiana 7, 141-147.

Miller, V.C. 1953. A quantitative geomorphic study of drainage basin characteristics inthe Clinch Mountain area, Virginia and Tennessee. Office of Naval Research, GeographyBranch, Project NR 389-042, Technical Report, 3, Columbia University.

Noto, L.V., La Loggia, G. 2009. Use of L-moments approach for regional flood frequency analysis in Sicily, Italy. Water Resources Management 23, 2207-2229. https://doi.org/10.1007/s11269-008-9378-x.

Poveda, G., Vélez J., Mesa O., Ceballos L., Zuluaga M. y Hoyos, C. 2002. Estimación de caudales mínimos para Colombia mediante regionalización y aplicación de la curva de recesión de caudales. Meteorológica Colombiana 6, 73-80.

Rusticucci, M., Zazulie, N., Raga G.B. 2014. Regional winter climate of the southern central Andes: Assessing the performance of ERA-Interim for climate studies. Journal of Geophysical Research Atmospheres 119, 8568-8582. https://doi.org/10.1002/2013JD021167

Schumm, S.A. 1956. Evolution of drainage systems and slopes in badlands at Perth Amboy, New Jersey. GSA Bulletin, 67 (5), 597-646. https://doi.org/10.1130/0016-7606(1956)67[597:EODSAS]2.0.CO;2

Seluchi, M.E., Garreaud, R.D., Norte, F.A., Saulo, A.C. 2006. Influence of the subtropical Andes on baroclinic disturbances: A cold front case study. Monthly Weather Review 134, 3317-3335. https://doi.org/10.1175/MWR3247.1

Song, J., Xia, J., Zhang, L., Wang, Z., Wan, H., She, D. 2016. Streamflow prediction in ungauged basins by regressive regionalization: a case study in Huai River Basin, China. Hydrology Research 47 (5). https://doi.org/10.2166/nh.2015.155

Teklu, T.H., Knut, A. 2017. Regional flood frequency analysis and prediction in ungauged basins including estimation of major uncertainties formid-Norway. Journal of Hydrology: Regional Studies 9, 104-126.

Témez, J.R. 1987. Cálculo hidrometeorológico de caudales máximos en pequeñas cuencas naturales. Dirección General de Carreteras, MOPU. 124 pp.

Tucci, C.E.M. 1997. Regionalização de vazões. $2^{\circ}$ ed. En: Hidrologia: Ciência e Aplicação. Tucci, C (Org). ABRH, Porto Alegre, p.573-620.

Viale, M., Bianchi, E., Cara, L., Ruiz, L., Villalba, R., Pitte, P., Masiokas, M., Rivera, J., Zalazar., L. 2019. Contrasting climates al both sides of the Andes in Argentina and Chile. Frontiers in Environmental Science 7 (69). http://doi.org/10.3389/fenvs.2019.00069. 
Vich, A.I.J., Norte, F.A., Lauro, C. 2014. Análisis regional de frecuencias de caudales de ríos pertenecientes a cuencas con nacientes en la Cordillera de los Andes. Meteorológica 39 (1), 3-26.

Vich, A.I.J. Norte, F. 2012. Análisis de frecuencias regional en cuencas con nacientes en la cordillera de Los Andes de Argentina. XI Congreso Argentino de Meteorología. CongreMet XI. Mendoza junio de 2012.

Vich, A.I.J. 1999. Aguas continentales. Formas y procesos. Manual de Aplicaciones prácticas. Mendoza. ZETA Editores.

Viglione, A., Claps P., Laio, F. 2007. Mean annual runoff estimation in North-Western Italy, In: G. Water resources assessment and management under water scarcity scenarios. La Loggia (Ed.). CDSU Publ. Milano. 